\title{
Recognizing Gynecological Cancer in Primary Care: Risk Factors, Red Flags, and Referrals
}

\author{
Garth Funston · Helena O'Flynn · Neil A. J. Ryan · Willie Hamilton • \\ Emma J. Crosbie
}

Received: December 14, 2017 / Published online: March 7, 2018

(C) The Author(s) 2018, corrected publication March 2018

\begin{abstract}
Early diagnosis of symptomatic gynecological cancer is likely to improve patient outcomes, including survival. The primary care practitioner has a key role to play in this-they must recognize the symptoms and signs of gynecological cancer and make prompt evidence-based decisions regarding further investigation and referral. However, this is often difficult as many of the symptoms of gynecological cancers are nonspecific and are more likely to be caused by benign rather than malignant disease. As primary care is generally the first point of patient contact, those working in this setting usually encounter cancer patients at an earlier, and
\end{abstract}

The original version of this article was revised due to a retrospective Open Access.

Enhanced content To view enhanced content for this article go to https://doi.org/10.6084/m9.figshare. 5914696.

G. Funston $(\bowtie) \cdot$ H. O'Flynn

Centre for Primary Care, University of Manchester, Manchester, UK

e-mail: gf272@doctors.org.uk

N. A. J. Ryan · E. J. Crosbie

Gynaecological Oncology Group, University of

Manchester, Manchester, UK

W. Hamilton

Exeter Medical School, University of Exeter, Exeter, UK possibly less symptomatic, stage than practitioners in secondary care. Despite these challenges, research has improved our understanding of the symptoms patients present to primary care with, and a range of tests and referral pathways now exist in the UK and other countries to aid early diagnosis. Primary care practitioners can also play a key role in gynecological cancer prevention. A significant proportion of gynecological cancer is preventable either through lifestyle changes such as weight loss, or, for cervical cancer, vaccination and/or engagement with screening programs. Primary care provides an excellent opportunity to discuss cancer risk with patients and to promote risk reduction strategies and lifestyle change. In this article, the first in a series discussing cancer detection in primary care, we concentrate on gynecological cancer and focus on the three most common forms that a primary care practitioner is likely to encounter: ovarian, endometrial, and cervical cancer. We outline key risk factors, briefly discuss prevention and screening strategies, and offer practical guidance on the recognition of symptoms and signs and the investigation and referral of women with suspected cancer. While this article is written from a UK primary care perspective, much of what is discussed will be of relevance to those working in other healthcare systems.

Keywords: Cervical cancer; Cancer detection; Cancer diagnosis; Cancer prevention; Cancer 
referrals; Endometrial cancer; General practice; Oncology; Ovarian cancer; Primary care

\section{OVARIAN CANCER}

\section{Background}

Ovarian cancer (OC) is the fifth most common cause of cancer death amongst women in the UK and USA, and over 7000 and 22,000 women are diagnosed, respectively, each year [1, 2]. Most OC symptoms are nonspecific, and the same symptoms occur more frequently in benign conditions. This makes OC particularly challenging for primary care practitioners to identify, and it is recognized as one of a group of cancers termed "harder to suspect" [3]. The majority of women are not diagnosed until the disease is advanced, which contributes to the UK's poor 5-year survival rate of $46 \%[1,4]$. Recognizing symptoms early, performing appropriate investigations, and referring promptly may improve patient outcomes $[5,6]$.

\section{Risk Factors and Epidemiology}

OC is predominantly a disease of the middle aged and elderly, with $75 \%$ of cancers occurring in women aged over 55 years [7]. Whilst most cases are sporadic, $5-15 \%$ are due to inherited cancer predisposition syndromes [8]. Mutations in BRCA1/BRCA2 are the most common inherited cause, and, while around $1.3 \%$ of women in the general population develop OC in their lifetime, $39 \%$ of female BRCA1 carriers will develop the disease [9]. Lynch syndrome, or hereditary non-polyposis colorectal cancer (HNPCC), is associated with a $6.7 \%$ lifetime risk of OC [10].

While the UK National Institute of Clinical Excellence (NICE) provides guidance for referral when concern exists about familial breast cancer risk [11], it does not provide equivalent guidance for OC. We recommend that the possibility of a hereditary cancer syndrome be considered in higher-risk patients, e.g., if multiple relatives have been diagnosed with OC or breast cancer (especially if diagnosed at a young age), if breast cancer and OC occur in the same relative, if a relative has been diagnosed with multiple cancers associated with Lynch syndrome such as gynecological, colorectal, gastrointestinal, or urological, or if the patient has a relative with a proven gene mutation. In such women, referral to a clinical geneticist may be warranted.

Lifestyle and environmental factors including obesity, long-term use of hormone replacement therapy, smoking, and asbestos exposure have all been linked to OC, and around $21 \%$ of ovarian cancers are thought to be "preventable" [8]. Factors that reduce the risk of OC include the oral contraceptive pill, childbirth, and breastfeeding.

\section{Screening}

Despite extensive research into OC screening, there is minimal evidence to support the implementation of costly national screening programs. The UK Collaborative Trial of Ovarian Cancer Screening (UKTOCS), which recruited over 200,000 women and evaluated both annual serum CA125 tests and annual transvaginal ultrasound, failed to show an overall reduction in OC mortality, although longer-term follow-up data is awaited [12].

\section{Symptoms and Signs}

Large case control studies have identified the symptoms with which patients are most likely to present in primary care $[13,14]$. The symptoms and signs recognized within current NICE guidelines are listed in Fig. 1. Symptoms such as bloating and pelvic pain are nonspecific and can be overlooked by doctors and patients alike [15]. Therefore, OC should be included in the differential diagnosis when assessing female patients with vague abdominopelvic symptoms. Recurrent or persistent symptoms may increase the likelihood of a malignant cause. Despite its previous reputation as a "silent disease" in early stages, most OC patients experience at least one symptom [16], and tend to experience more, with a higher frequency and at greater severity than patients with benign disease [13]. 


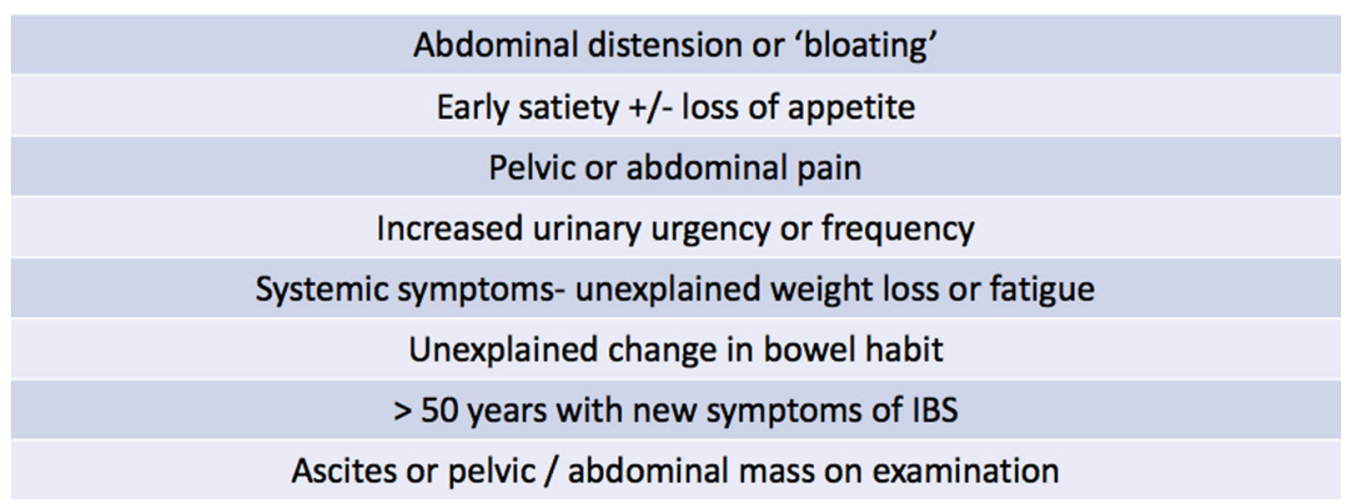

Fig. 1 Symptoms and signs of ovarian cancer. Based on 2015 NICE guidelines for suspected cancer recognition and referral

One presentation that warrants special consideration is the new onset of irritable bowel syndrome (IBS) in patients over 50 years of age. There is significant overlap in the symptom profiles of OC and IBS and misattributing nonspecific abdominal symptoms to IBS may contribute to a delayed OC diagnosis [17]. A new presentation of apparent IBS in women over 50 years of age is unusual and should prompt investigation for OC.

NICE guidelines on the investigation and referral of OC are summarized in Fig. 2. Although NICE does not give hard age cutoffs, it does recognize that women over the age of 50 years are at increased risk [18]. While the identification of a pelvic or abdominal mass on examination is an immediate cause for concern and warrants an urgent referral via the suspected cancer pathway, investigations for other presentations can be safely initiated within primary care.

\section{Investigation}

CA125 is the NICE-advocated first-line investigation for OC, and, if CA125 is elevated (> $35 \mathrm{IU} / \mathrm{ml})$, NICE recommends an outpatient abdominopelvic ultrasound (US) scan. Transvaginal US is generally the preferred method of US as it allows for more detailed images of the ovaries to be obtained when compared with transabdominal US [19].

Debate exists regarding the most appropriate approach for the investigation of OC symptoms in primary care. CA125 provides a simple, cheap, and convenient initial test for OC; however, it is far from perfect. It is elevated in many common benign conditions, including fibroids and endometriosis, and tends to be higher in younger patients. It has limited sensitivity, particularly in early stage disease, where

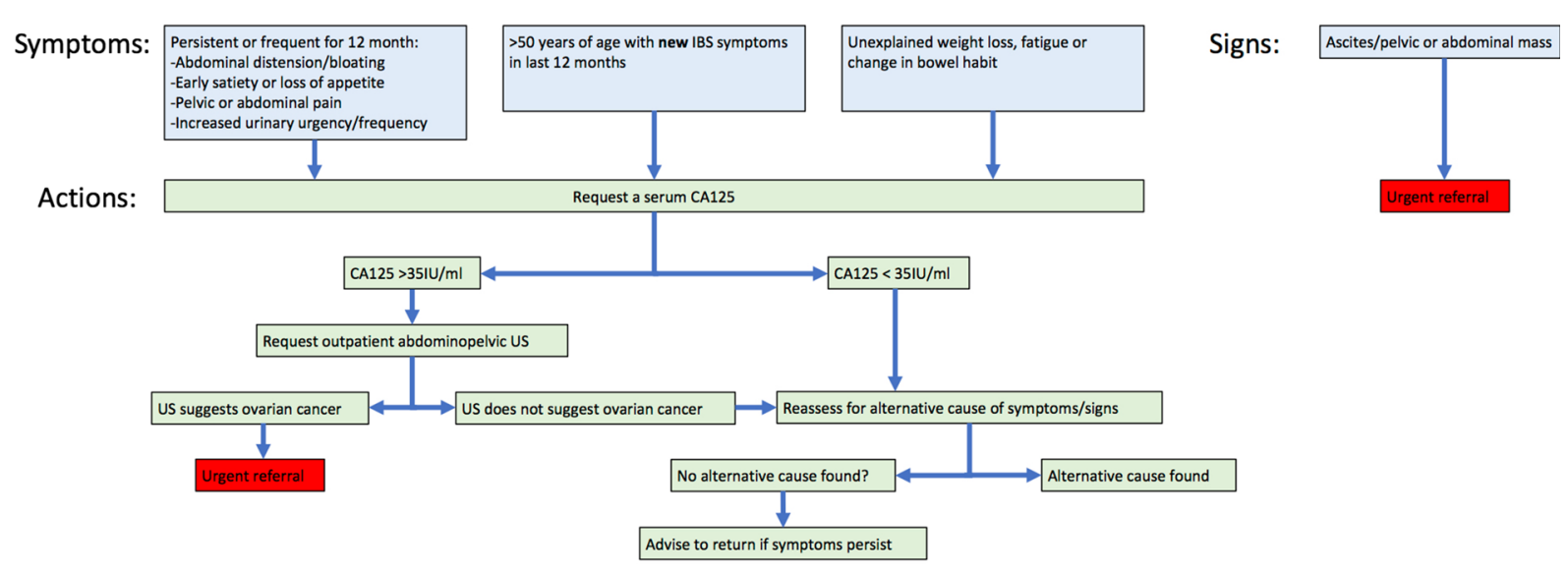

Fig. 2 Investigation and referral of suspected ovarian cancer in primary care. Based on 2015 NICE guidelines for suspected cancer recognition and referral 
it is elevated in only $50 \%$ of patients [7]. What is more, the threshold of $>35 \mathrm{IU} / \mathrm{ml}$ for an abnormal result is not based on primary care data. Other countries recommend alternative approaches for initial investigation. For example, Scottish guidelines advocate ordering an US alongside CA125 [20]. Regardless of which approach is taken, requesting tests early and acting on results promptly is likely to be beneficial, and even if the explanation is benign, the woman has been reassured at an early stage.

A situation that causes uncertainty amongst primary care practitioners is what action to take in women with an elevated CA125 and a normal US. While NICE guidance simply advises that other causes for the symptoms be sought and that the patient be advised to return if the symptoms persist, debate exists surrounding this guidance [21]. A recent study found that $80 \%$ of GPs deviate from NICE guidance and refer this group of patients to secondary care [22]. A pragmatic approach, and one advocated in Republic of Ireland guidelines [23], is to monitor the CA125 level in primary care, e.g., performing a repeat blood test at 6 weeks. However, this approach is not evidence based.

As CA125 is elevated in benign and physiological conditions, it is important to have a clear rationale for testing and to counsel patients carefully regarding the potential implications of testing. This is perhaps particularly relevant in young patients with a low risk of OC but a high risk of false positive results. Having said this, in our experience patients are generally happy to undergo CA125 testing and evidence suggests that patients often set a lower risk threshold for cancer investigations than that set by UK guidelines [24]. Reassuringly, symptom-triggered testing using CA125 and US appears to result in little unnecessary surgery [25].

\section{Summary}

Identifying $\mathrm{OC}$ is a real challenge for primary care practitioners, yet early recognition may improve patient outcomes. Assessment should include family history, a comprehensive symptom enquiry, and abdominopelvic examination. OC should be included in the differential diagnosis when assessing women with nonspecific abdominopelvic symptoms, particularly those over the age of 50 years. While there is no ideal test for ovarian cancer, CA125 and US both provide reasonable options.

\section{ENDOMETRIAL CANCER}

\section{Background}

Endometrial cancer (EC) is the fourth most common cancer affecting women and the most common form of gynecological cancer in the developed world, with over 9000 and 61,000 women diagnosed in the UK and USA, respectively, each year $[26,27]$. The incidence has risen by more than $50 \%$ over the past two decades [26]. Disease-specific survival is heavily stage dependant with 5-year survivals of 95\% and $14 \%$ for women diagnosed in stages I and IV, respectively [28]. Therefore, the expedited diagnosis of uterine cancer is crucial; indeed early diagnosis is believed to be of greater benefit than for the majority of other cancers [5]. While postmenopausal bleeding (PMB) is the classical "red flag symptom", several other symptoms and signs can also herald the disease.

\section{Risk Factors and Epidemiology}

EC is strongly related to age, with $75 \%$ of diagnoses occurring in women above the age of 55 years [29]. Other risk factors include early menarche, late menopause, nulliparity, tamoxifen use, and polycystic ovary syndrome. The majority of cases are sporadic, but around 10\% result from genetic syndromes such as Lynch syndrome [30]. Most risk factors for sporadic EC exert their influence through increasing estrogen exposure without a compensatory increase in progesterone. Conversely, the combined oral contraceptive pill, which reduces unopposed estrogen exposure, reduces the risk of EC [31].

The most important modifiable risk factor for EC is excess body weight. No other cancer is more strongly associated with obesity [32, 33], which underpins over a third of ECs in the UK [34]. Along with increased life expectancy, the 
rise in obesity in recent decades likely accounts for the rise in endometrial cancer incidence [35]. The principal mechanism behind this association is increased peripheral conversion of androgens to estrogen in adipose tissue. In addition, increased adiposity leads to insulin resistance and a pro-inflammatory environment, both of which have been causally linked with EC [36]. Type 2 diabetes has previously been reported as an independent risk factor for EC, but obesity is a major confounder [37].

Whilst there are many risk factors for EC, most are unlikely to affect a primary care practitioner's decision to investigate or refer. However, a raised BMI, a strong personal, or family history of Lynch syndrome-associated cancers and current or previous use of tamoxifen should increase clinical suspicion.

\section{Screening}

There is no evidence for population-level screening for EC. Ultrasound, the most commonly investigated screening method, has not been shown to reduce mortality [38], and current serum biomarkers lack the necessary sensitivity and specificity. Some high-risk women, such as those with Lynch syndrome, may be offered screening, although its application is not uniform nor the screening methods consistent. Even in this high-risk group evidence that screening improves survival is lacking [39].

\section{Symptoms and Signs}

The classic red flag symptom of postmenopausal bleeding, unexplained vaginal bleeding more than 12 months after menopausal amenorrhea, is observed in up to $90 \%$ of presentations in secondary care [35], and $4 \%$ of those who present in primary care with postmenopausal bleeding have EC [40]. As such, an urgent referral should be considered in all patients presenting with this symptom. Around onethird of UK women who present to their GP with postmenopausal bleeding are not immediately referred, although the reasons for these delays are poorly understood [41].
Other features associated with EC are abnormal vaginal bleeding (heavy or irregular menstrual bleeding) in premenopausal patients, vaginal discharge, hematuria, anemia, thrombocytosis, raised blood glucose, and abdominal pain [40]. These features, save for abdominal pain, are included in current NICE guidelines [18]. As such, investigation of patients with suspected EC should include a full blood count and blood glucose level, in addition to tests to exclude alternative diagnoses, e.g., vaginal swabs and a clotting profile. However, these investigations should not delay urgent referral where indicated. Primary care case control studies have demonstrated that combinations of presenting features, e.g. hematuria and high blood glucose in an over 55-year-old patient, significantly increase the risk that a patient has EC, when compared to the presence of a single presenting feature [40]. This accounts for the counterintuitive combinations of symptoms/ signs in NICE guidance recommended to trigger an outpatient US (Fig. 3).

As EC risk is strongly related to age, NICE guidelines include specific age cutoffs for several referral and investigation criteria, as outlined in Fig. 3. Even so, it is worth keeping in mind that $15 \%$ of EC cases in the UK occur in those below the age of 55 years [29], and intermenstrual bleeding or vaginal discharge in this group should not be attributed to benign pathology without considering sinister causes. Investigation should include vaginal swabs to exclude infection and an US if symptoms persist. In contrast to NICE guidance, Scottish guidelines do not include age-specific cutoffs [20], and the American College of Obstetrics and Gynecologists advocate investigation for possible EC in those over 45 years of age or in younger patients with risk factors for unopposed estrogen exposure [42].

While physical examination findings in EC, such as pelvic masses, are late signs, an abdominopelvic examination is important as it may identify alternative causes for the patients' symptoms, such as cervical lesions.

As poor patient awareness of red flags is associated with delayed presentation, opportunistic patient education on key symptoms, e.g., during discussions about menopause or 


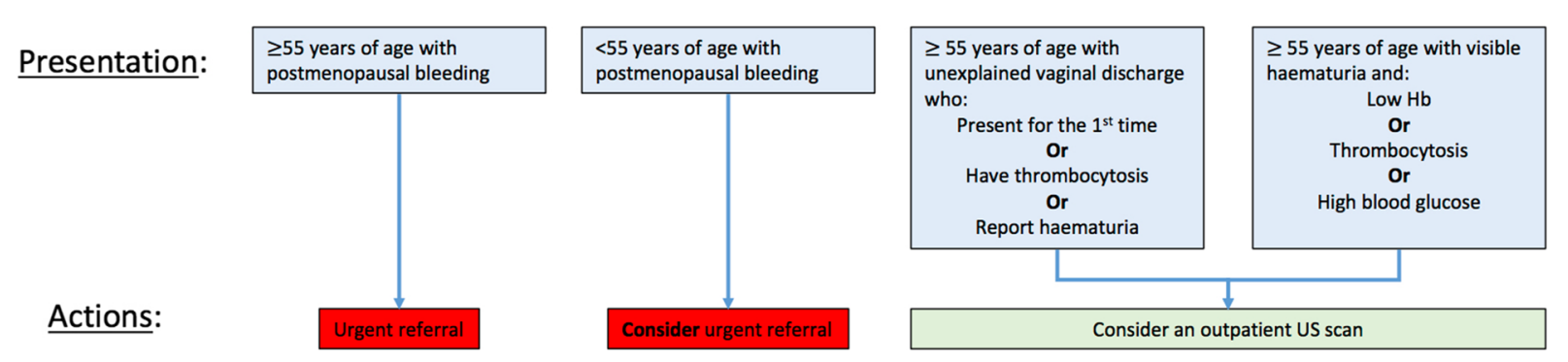

Fig. 3 Investigation and referral of suspected endometrial cancer in primary care. Based on 2015 NICE guidelines for suspected cancer recognition and referral

hormone replacement therapy, may play a role in preventing delays in health-seeking behavior $[43,44]$.

\section{Investigations}

In the UK, NICE guidance recommends a pelvic US in women aged 55 years and over with unexplained vaginal discharge who are either presenting for the first time or have thrombocytosis or hematuria. The same advice applies to those attending with visible hematuria who are either anemic or have thrombocytosis or hyperglycemia on investigation. In symptomatic postmenopausal women, an endometrial thickness of greater than $4 \mathrm{~mm}$ on US should prompt urgent referral [45]. Of note, data from the UK Collaborative Trial of Ovarian Cancer Screening (UKCTOCS) indicates that an incidentally detected endometrial thickness of greater than $10 \mathrm{~mm}$ in asymptomatic postmenopausal women should also prompt further investigation [38]. For premenopausal women, the interpretation of US is much more challenging because of the natural fluctuations in endometrial thickness during the menstrual cycle.

\section{Summary}

EC is the most common gynecological cancer encountered in primary care and the incidence has increased dramatically as a result of improved life expectancies and the rise in obesity. Educating patients about key warning signs and acting on symptoms and signs in a timely manner is vital, as, if diagnosed early, EC has an excellent prognosis.

\section{CERVICAL CANCER}

\section{Background}

In contrast to $\mathrm{OC}$ and $\mathrm{EC}$, invasive cervical cancer (CC) has become largely preventable in developed countries, through the introduction of effective screening and vaccination programs. However, internationally, mortality remains high [46]. In the UK, over 3000 women are diagnosed each year with $10 \%$ of diagnoses occurring in an emergency setting, which is associated with poorer outcomes [47]. Furthermore, over 30\% of women with CC are referred into secondary care via routine non-cancer pathways, which can result in delayed diagnosis [48]. While in recent years much progress has been made in the effective screening and management of premalignant lesions, there is still a need to ensure public awareness of the symptoms of overt CC. An understanding of the disease, how it can be prevented through vaccination, and how to evaluate symptomatic patients is important for those working in primary care.

\section{Epidemiology and Pathology}

Worldwide, 527,000 new cases were recorded in 2012 [49]. Internationally over 250,000 CC-related deaths occur per year, which are mainly attributable to the lack of effective vaccination and screening programs in developing countries 
with scarce resources [50]. In the UK, incidence is highest in women between the ages of 25 and 45 years, with a second peak in $85-89$ years old [51]. Increased incidence rates are observed in areas of higher socioeconomic deprivation within the UK [52].

The majority of premalignant and malignant lesions develop within the squamocolumnar junction through viral neoplastic transformation [53]. Dysplastic changes begin in the form of cervical intraepithelial neoplasia (CIN) and adenocarcinoma in situ, which can take several years to develop into invasive cancer. Such a time lag creates the potential for effective screening and management of precancerous lesions through coordinated national programs.

CIN is graded as 1,2 , and 3 with increasing severity, depending on the proportion of epithelium demonstrating dysplastic changes. CIN1 commonly regresses spontaneously. CIN 2 and 3, together referred to as "high grade CIN", have a higher rate of malignant transformation, and treatment is advocated [54].

\section{Risk Factors}

Human papillomavirus (HPV) is a common infection associated with genital skin to skin contact. Most women will have a transient infection only. Persistent infections are associated with integration of HPV DNA into the host cell genome, giving rise to premalignant, and, less frequently, malignant change [53]. Highrisk HPV (hrHPV) types 16 and 18 are together responsible for around 70\% of CC worldwide while the remainder of CC cases are associated with other high-risk types [55].

Use of the combined hormonal contraception and tobacco smoking are associated with an increased risk of CC $[56,57]$. Patients with HIV have a sixfold increase in their risk of developing CC, but this decreases with HIV treatment [58]. Co-infection with Chlamydia trachomatis and persistent infection with lowrisk HPV types may also increase the risk of CC [57].

Despite public awareness campaigns, many women have a limited understanding of HPV, its mode of transmission, and its link to various cancers [59]. Primary care practitioners have reported that, in addition to lacking in-depth knowledge about HPV, they find discussions regarding HPV particularly challenging $[60,61]$. To facilitate these discussions, evidence-based consultation guides have recently been developed [61].

\section{Symptoms and Signs}

Most women with premalignant lesions are asymptomatic. Features associated with invasive cervical cancer include intermenstrual, postcoital, and postmenopausal bleeding, persistent vaginal discharge, hematuria, urinary tract infection, abdominal pain, high white cell count, and low hemoglobin [62, 63]. As discussed, postmenopausal bleeding is of particular concern and practitioners should consider a referral on the suspected cancer pathway. Although symptoms such as vaginal discharge or intermenstrual bleeding are less strongly predictive of cancer, they should not be discounted without further evaluation. Figure 4 outlines our suggested diagnostic workup for these patients.

Of note, lack of recognition of concerning symptoms among patients, e.g., vaginal discharge in younger patients (under 25 years of age), is likely to contribute to delayed diagnosis [64].

Women reporting high-risk symptoms should undergo an adequate genital examination with visualization of the cervix and a full diagnostic workup including an infection screen. In one study, only $39 \%$ of patients who were referred and subsequently diagnosed with CC had a documented cervical examination in primary care [65]. While a visualized lesion is obviously an important finding and warrants urgent referral on a suspected cancer pathway, a normal cervical exam does not exclude cervical cancer, which can be microscopic at presentation.

A useful algorithm for the management of young patients with worrisome symptoms has been developed by the UK Department of Health [66]. Cervical cytology may miss invasive cancer and is not useful as a diagnostic test 


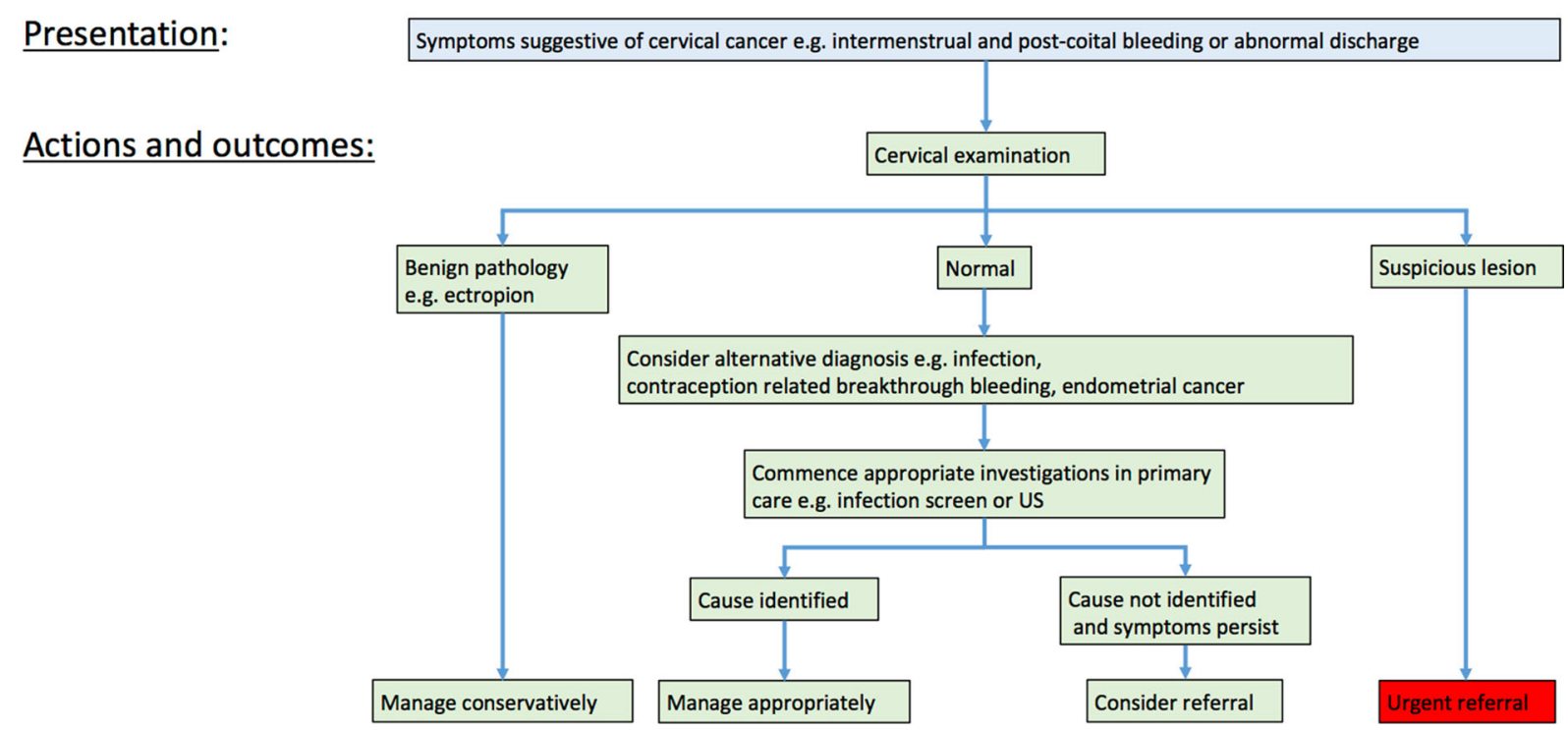

Fig. 4 Suggested management of patients presenting to primary care with cervical cancer symptoms. GPs should maintain a high index of suspicion in those with an incomplete screening history

in symptomatic patients; thus, referral should never be delayed to await a smear result. Recent evidence, however, indicates that cervical cytology can be useful to "rule in" women for urgent referral [67].

\section{Screening Programs}

In the UK, the cervical screening program is offered 3-yearly to all women between the ages of 25 and 49 and then at 5-yearly intervals until the age of 64 . Screening uptake is $70-73 \%$ across the UK [68]. Since 2013, HPV triage has been adopted to select women with borderline or mild dyskaryotic changes for colposcopy if they are positive for hrHPV. This test has high sensitivity [69]. All women with CIN who have undergone treatment are offered a "test of cure" in the community at 6 months. Those with borderline or low grade dyskaryosis who are hrHPV negative return to routine 3-years cytology recall. The use of primary hrHPV screening is currently being rolled out across England [70]. The advantage of hrHPV testing over cytology as the initial screening test is its high negative predictive value, which could allow women who are hrHPV negative to have an extended interval between screens [71].
A woman requesting cervical screening at an earlier age is common in primary care. Such women should be counselled on the rationale of screening age cutoffs, including potentially unnecessary treatments, spontaneous regression of CIN1, and increased risk of preterm delivery after treatment of the cervix. CC is rare in women under the age of 25 years and screening does not improve survival in this select group. Specific guidance is available on protocols for smear tests in women post hysterectomy with previous CIN treatment, HIVpositive patients, those in the postnatal period, and for transgender patients [70].

Women with moderate or high grade dyskaryosis are offered colposcopy through the screening agency, as are those with hrHPVpositive minor cytological abnormalities. Work is ongoing to triage women with hrHPV even more accurately using molecular and immunohistochemistry tests [72]. Depending on colposcopic results, a directed biopsy is taken or a "see and treat" approach offered, generally in the form of large loop excision of the transformation zone (LLETZ). Recent data implicating large or repeated LLETZ excisions as risk factors for mid-trimester miscarriage or preterm birth have brought about a return to nondestructive ablative techniques, particularly cold coagulation, 
for treatment of CIN2 lesions, as these are less associated with poor obstetric outcomes [73].

\section{Vaccination Program}

The HPV vaccination program has been available in the UK since 2008 and is routinely offered in secondary schools at ages 12 or 13 . The quadrivalent vaccine, protecting against HPV 16, 18, and low-risk genital wart types 6 and 11 , has replaced the bivalent vaccine as the preferred choice; it is given twice, at least 6 months and less than 12 months apart. Vaccination practice varies internationally; for example, in the USA, vaccination of boys is advocated to prevent oropharyngeal, anal, and penile cancers [53]. Significant reductions in HPV rates have been observed in vaccinated as well as unvaccinated populations, confirming the development of herd immunity [74].

Despite reports in the media which have questioned the safety of HPV vaccination programs, parents should be advised that there are few serious adverse events related to the vaccine and that no association between the vaccine and chronic illness has been identified [50, 75].

\section{Summary}

Screening and vaccination programs have significantly reduced the incidence of $\mathrm{CC}$, and should be advocated by primary care practitioners. Despite these programs, thousands of CCs are diagnosed each year in the UK alone, mostly in women who do not regularly attend screening. Symptomatic presentations still occur and primary care practitioners should ensure that patients with persistent abnormal bleeding or discharge are examined and referred appropriately.

\section{CONCLUSION}

Our understanding of how patients with cancer present in primary care, including patients with OC-a disease once considered asymptomatic in the early stages-has increased significantly in recent years. Despite this, early detection of cancer poses challenges for those working in general practice. As a result of the nonspecific nature of many of the symptoms of gynecological cancers, primary care practitioners are likely to see patients who "could have cancer" virtually every day, though the vast majority of these patients will not have cancer. It is not feasible or appropriate to refer all patients who present with vague symptoms, such as bloating, for scans or further investigation. However, an awareness of risk factors and key presenting symptoms and signs enables practitioners to make evidence-based decisions on who to investigate in primary care, who to refer, and on the urgency of those referrals.

Primary care practitioners have a wide variety of tools at their disposal to investigate the "could have cancer" patient, from serum biomarkers to imaging techniques. The number and complexity of these tools are likely to increase as technology advances. Yet, no test is perfect and as false positive results can lead to unnecessary investigations, referrals, and patient distress, they should be used judiciously. In the UK, the urgent referral pathway for suspected cancer cases, or "2-week wait pathway", offers an excellent opportunity for patients to be reviewed rapidly and has been linked to better patient outcomes [76]. Despite this, many women with gynecological cancers are still referred via routine pathways.

The role of primary care in cancer prevention, through advocating lifestyle change, vaccination, and screening, has its own challenges. Yet, engaging patients in discussions about cancer risk and encouraging and supporting change have an important role to play in cancer prevention.

\section{ACKNOWLEDGEMENTS}

Funding. No funding was obtained to support this work.

Authorship. All named authors meet the International Committee of Medical Journal Editors (ICMJE) criteria for authorship for this manuscript, take responsibility for the integrity 
of the work as a whole, and have given final approval for the version to be published.

Disclosures. Garth Funston, Helena O'Flynn, Neil A.J. Ryan, Willie Hamilton, and Emma J. Crosbie have nothing to disclose.

Compliance with Ethical Guidelines. This article is based on previously conducted studies and does not involve any new studies of human or animal subjects performed by any of the authors.

Open Access. This article is distributed under the terms of the Creative Commons Attribution-NonCommercial 4.0 International License (http://creativecommons.org/licenses/ by-nc/4.0/), which permits any noncommercial use, distribution, and reproduction in any medium, provided you give appropriate credit to the original author(s) and the source, provide a link to the Creative Commons license, and indicate if changes were made.

\section{REFERENCES}

1. Cancer Research UK. Ovarian cancer mortality statistics. http://www.cancerresearchuk.org/healthprofessional/cancer-statistics/statistics-by-cancertype/ovarian-cancer/mortality. Accessed July 26, 2017.

2. United States Centres for Disease Control and Prevention. Ovarian cancer statistics. https://www. cdc.gov/cancer/ovarian/statistics/index.htm. Accessed July 26, 2017.

3. Lyratzopoulos G, Wardle J, Rubin G. Rethinking diagnostic delay in cancer: how difficult is the diagnosis? BMJ. 2014;349:g7400.

4. Maringe C, Walters S, Butler J, et al. Stage at diagnosis and ovarian cancer survival: evidence from the international cancer benchmarking partnership. Gynecol Oncol. 2012;127:75-82.

5. Hamilton W, Stapley S, Campbell C, et al. For which cancers might patients benefit most from expedited symptomatic diagnosis? Construction of a ranking order by a modified Delphi technique. BMC Cancer. 2015;15:820.

6. Neal RD, Tharmanathan $\mathrm{P}$, France $\mathrm{B}$, et al. Is increased time to diagnosis and treatment in symptomatic cancer associated with poorer outcomes? Syst Rev Br J Cancer. 2015;112:S92-107.

7. Sundar S, Neal R, Kehoe E. Diagnosis of ovarian cancer. BMJ. 2015;351:h5543.

8. Cancer Research UK. Ovarian cancer risk factors. http://www.cancerresearchuk.org/health-professio nal/cancer-statistics/statistics-by-cancer-type/ovari an-cancer/risk-factors\#heading-Eleven. Accessed July 26, 2017.

9. National Cancer Institute. BRCA1 and BRCA2: cancer risk and genetic testing. http://www.cancer. gov/about-cancer/causes-prevention/genetics/brcafact-sheet\#r4. Accessed July 26, 2017.

10. Watson P, Vasen HFA, Mecklin JP, et al. The risk of extra-colonic, extra-endometrial cancer in the Lynch syndrome. Int J Cancer. 2008;123:444-9.

11. National Institute of Clinical Excellence. Familial breast cancer: classification, care and managing breast cancer and related risks in people with a family history of breast cancer. http://www.nice. org.uk/guidance/cg164. Accessed September 29, 2017.

12. Jacobs IJ, Menon U, Ryan A, et al. Ovarian cancer screening and mortality in the UK collaborative trial of ovarian cancer screening (UKCTOCS): a randomised controlled trial. Lancet. 2016;387:945-56.

13. Goff BA, Mandel LS, Melancon CH, Muntz HG. Frequency of symptoms of ovarian cancer in women presenting to primary care clinics. JAMA. 2004;291:2705-12.

14. Hamilton W, Peters TJ, Bankhead C, Sharp D. Risk of ovarian cancer in women with symptoms in primary care: population based case-control study. BMJ. 2009;339:b2998.

15. Bankhead CR, Collins C, Stokes-Lampard H, et al. Identifying symptoms of ovarian cancer: a qualitative and quantitative study. BJOG. 2008;115:1008-14.

16. Vine MF, Calingaert B, Berchuck A, Schildkraut JM. Characterization of prediagnostic symptoms among primary epithelial ovarian cancer cases and controls. Gynecol Oncol. 2003;90:75-82.

17. Goff BA, Mandel L, Muntz HG, Melancon $\mathrm{CH}$. Ovarian carcinoma diagnosis. Cancer. 2000;89:2068-75.

18. National Institute of Clinical Excellence. Suspected cancer: recognition and referral. http://www.nice. org.uk/guidance/ng12. Accessed July 26, 2017. 
19. Fischerova D. Ultrasound scanning of the pelvis and abdomen for staging of gynecological tumors: a review. Ultrasound Obstet Gynecol. 2011;38:246-66.

20. Health Improvement Scotland. Scottish referral guidelines for suspected cancer. http://www.health careimprovementscotland.org/our_work/cancer_ care_improvement/programme_resources/scottish_ referral_guidelines.aspx. Accessed September 29, 2017.

21. National Institute of Clinical Excellence. 4-year surveillance 2016- ovarian cancer (2011) NICE guideline CG122, Appendix A. https://www.nice.org. $\mathrm{uk} /$ guidance/cg122/resources/surveillance-report2016-ovarian-cancer-2011-nice-guideline-cg1223073930701253. Accessed September 29, 2017.

22. Moss EL, Moran A, Reynolds TM, Stokes-Lampard $\mathrm{H}$. Views of general practitioners on the role of CA125 in primary care to diagnose ovarian cancer. BMC Womens Health. 2013;13:8.

23. Ireland National Cancer Control Programme. Ovarian cancer GP referral for symptomatic women. http://www.hse.ie/eng/services/list/5/ cancer/profinfo/resources/gpreferrals/Ovarian-CancerReferral-Guidelines.pdf. Accessed December 2, 2017.

24. Banks J, Hollinghurst S, Bigwood L, et al. Preferences for cancer investigation: a vignette-based study of primary-care attendees. Lancet Oncol. 2014;15:232-40.

25. Andersen MR, Lowe KA, Goff BA. Value of symptom-triggered diagnostic evaluation for ovarian cancer. Obstet Gynecol. 2014;123:73-9.

26. Cancer Research UK. Uterine cancer statistics. http://www.cancerresearchuk.org/health-professional/ cancer-statistics/statistics-by-cancer-type/uterinecancer\#heading-Zero. Accessed September 29, 2017.

27. US National Cancer Institute. Cancer stat facts: endometrial cancer. https://seer.cancer.gov/ statfacts/html/corp.html. Accessed July 26, 2017.

28. Cancer Research UK. Uterine cancer survival statistics. http://www.cancerresearchuk.org/healthprofessional/cancer-statistics/statistics-by-cancertype/uterine-cancer/survival\#heading-Three. Accessed July 26, 2017.

29. Cancer Research UK. Uterine cancer statistics. http://www.cancerresearchuk.org/health-professional/ cancer-statistics/statistics-by-cancer-type/uterinecancer\#heading-Three. Accessed September 29, 2017.
30. Okuda T, Sekizawa A, Purwosunu Y, et al. Genetics of endometrial cancers. Obstet Gynecol Int. 2010;2010:1-8.

31. Maxwell GL, Schildkraut JM, Calingaert B, et al. Progestin and estrogen potency of combination oral contraceptives and endometrial cancer risk. Gynecol Oncol. 2006;103:535-40.

32. Renehan AG, Tyson M, Egger M, Heller RF, Zwahlen M. Body-mass index and incidence of cancer: a systematic review and meta-analysis of prospective observational studies. Lancet. 2008;371:569-78.

33. Bhaskaran K, Douglas I, Forbes H, et al. Body-mass index and risk of 22 specific cancers: a populationbased cohort study of 5.24 million UK adults. Lancet. $2014 ; 384: 755-65$.

34. Cancer Research UK. Uterine cancer risk factors. http://www.cancerresearchuk.org/health-professional/ cancer-statistics/statistics-by-cancer-type/uterinecancer/risk-factors\#heading-Eight. Accessed September 29, 2017.

35. Amant F, Moerman P, Neven P, et al. Endometrial cancer. Lancet. 2005;366:491-505.

36. Nieman KM, Romero IL, Van Houten B, Lengyel E. Adipose tissue and adipocytes support tumorigenesis and metastasis. Biochim Biophys Acta Mol Cell Biol Lipids. 2013;1831:1533-41.

37. Luo J, Beresford S, Chen C, et al. Association between diabetes, diabetes treatment and risk of developing endometrial cancer. $\mathrm{Br} \mathrm{J}$ Cancer. 2014;111:1432-9.

38. Jacobs I, Gentry-Maharaj A, Burnell M, et al. Sensitivity of transvaginal ultrasound screening for endometrial cancer in postmenopausal women: a case-control study within the UKCTOCS cohort. Lancet Oncol. 2011;12:38-48.

39. Lindor NM, Petersen GM, Hadley DW, et al. Recommendations for the care of individuals with an inherited predisposition to Lynch syndrome. JAMA. 2006;296:1507.

40. Walker S, Hyde C, Hamilton W. Risk of uterine cancer in symptomatic women in primary care: case-control study using electronic records. $\mathrm{Br} \mathrm{J}$ Gen Pract. 2013;63:643-8.

41. McBride D, Hardoon S, Walters K, Gilmour S, Raine R. Explaining variation in referral from primary to secondary care: cohort study. BMJ. 2010;341:c6267.

42. ACOG. CO557: Management of acute abnormal uterine bleeding in nonpregnant reproductive-aged women. Obstet Gynecol. 2013;121:891-6. 
43. Niksic M, Rachet B, Warburton FG, et al. Cancer symptom awareness and barriers to symptomatic presentation in England-are we clear on cancer? $\mathrm{Br}$ J Cancer. 2015;113:533-42.

44. Power E, Wardle J. Change in public awareness of symptoms and perceived barriers to seeing a doctor following be clear on cancer campaigns in England. Br J Cancer. 2015;112:S22-6.

45. Colombo N, Preti E, Landoni F, et al. Endometrial cancer: ESMO clinical practice guidelines for diagnosis, treatment and follow-up. Ann Oncol. 2011;22:35-9.

46. Sankaranarayanan R. Screening for cancer in lowand middle-income countries. Ann Glob Health. 2014;80:412-7.

47. Zhou Y, Ga A, Hamilton W, et al. Defining, measuring and preventing the diagnosis of cancer as an emergency: a critical review of current evidence. Nat Publ Gr. 2016;14:1-35.

48. National Cancer Intellegence Network. Routes to diagnosis 2006-2013 workbook (b). http://www. ncin.org.uk/publications/routes_to_diagnosis. Accessed September 29, 2017.

49. Ferlay J, Soerjomataram I EM. Cancer Incidence and Mortality Worldwide: IARC CancerBase No. 11. Lyon, France: International Agency for Research on Cancer.

50. World Health Organisation. WHO guidelines for treatment of cervical intraepithelial neoplasia 2-3 and adenocarcinoma in situ: cryotherapy, large loop excision of the transformation zone, and cold knife conization. http://www.who.int/reproductive health/publications/cancers/screening_and_treatment _of_precancerous_lesions/en/index.html. Accessed July 26, 2017.

51. Cancer Research UK. Cervical cancer incidence statistics. http://www.cancerresearchuk.org/healthprofessional/cancer-statistics/statistics-by-cancer-type/ cervical-cancer/incidence. Accessed July 26, 2017.

52. National Cancer Intellegence Network. Cancer by deprivation in England incidence, 1996-2010, Mortality, 1997-2011. http://www.ncin.org.uk/ about_ncin/cancer_by_deprivation_in_england. Accessed September 29, 2017.

53. Small W, Bacon MA, Bajaj A, et al. Cervical cancer: a global health crisis. Cancer. 2017;123:2404-12.

54. Massad LS, Einstein MH, Huh WK, et al. 2012 updated consensus guidelines for the management of abnormal cervical cancer screening tests and cancer precursors. J Low Genit Tract Dis. 2013;17:S1-27.
55. Crosbie EJ, Einstein MH, Franceschi S, Kitchener HC. Human papillomavirus and cervical cancer. Lancet. 2013;382:889-99.

56. International Collaboration of Epidemiological Studies of Cervical Cancer, Appleby P, Beral V, et al. Cervical cancer and hormonal contraceptives: collaborative reanalysis of individual data for 16,573 women with cervical cancer and 35,509 women without cervical cancer from 24 epidemiological studies. Lancet. 2007;370:1609-21.

57. Cancer Research UK. Cervical cancer statistics. http://www.cancerresearchuk.org/health-professional/ cancer-statistics/statistics-by-cancer-type/cervicalcancer/risk-factors. Accessed September 27, 2017.

58. Blitz S, Baxter J, Raboud J, et al. Evaluation of HIV and highly active antiretroviral therapy on the natural history of human papillomavirus infection and cervical cytopathologic findings in HIV-positive and high-risk HIV-negative women. J Infect Dis. 2013;208:454-62.

59. Low EL, Simon AE, Lyons J, Romney-Alexander D, Waller J. What do British women know about cervical cancer symptoms and risk factors? Eur J Cancer. 2012;48:3001-8.

60. McSherry LA, Dombrowski SU, Francis JJ, et al. "It"s a can of worms': understanding primary care practitioners' behaviours in relation to HPV using the theoretical domains framework. Implement Sci. 2012;7:73.

61. Hendry M, Pasterfield D, Gollins S, et al. Talking about human papillomavirus and cancer: development of consultation guides through lay and professional stakeholder coproduction using qualitative, quantitative and secondary data. BMJ Open. 2017;7:e015413.

62. Walker S, Hamilton W. Risk of cervical cancer in symptomatic women aged $\geq 40$ in primary care: a case-control study using electronic records. Eur J Cancer Care (Engl). 2017. https://doi.org/10.1111/ ecc.12706.

63. Stapley S, Hamilton W. Gynaecological symptoms reported by young women: examining the potential for earlier diagnosis of cervical cancer. Fam Pract. 2011;28:592-8.

64. Lim AW, Ramirez AJ, Hamilton W, et al. Delays in diagnosis of young females with symptomatic cervical cancer in England: an interview-based study. Br J Gen Pract. 2014;64:e602-10.

65. Lim AWW, Sasieni P, Hamilton W, Stapley S, Hollingworth A. Performance characteristics of visualising the cervix in symptomatic young females: a review of primary care records in females 
with and without cervical cancer. Br J Gen Pract. 2016;66:e189-92.

66. UK Department of Health. Clinical practice guidelines for the assessment of young women aged 20-24 with abnormal vaginal bleeding. https:// www.gov.uk/government/uploads/system/uploads/ attachment_data/file/436924/doh-guidelines-youngwomen.pdf. Accessed July 26, 2017.

67. Lim AW, Landy R, Castanon A, et al. Cytology in the diagnosis of cervical cancer in symptomatic young women: a retrospective review. $\mathrm{Br} \mathrm{J}$ Gen Pract. 2016;66:e871-9.

68. Cancer Research UK. Cervical cancer diagnosis and treatment statistics. http://www.cancerresearchuk. org/health-professional/cancer-statistics/statisticsby-cancer-type/cervical-cancer/diagnosis-and-treat ment. Accessed September 29, 2017.

69. Kelly RS, Patnick J, Kitchener HC, Moss SM. HPV testing as a triage for borderline or mild dyskaryosis on cervical cytology: results from the Sentinel Sites study. Br J Cancer. 2011;105:983-8.

70. Public Health England. NHS cervical screening programme: colposcopy and programme management. 3rd ed. London: Public Health England; 2016.
71. Ronco G, Dillner J, Elfström KM, et al. Efficacy of HPV-based screening for prevention of invasive cervical cancer: follow-up of four European randomised controlled trials. Lancet. 2014;383:524-32.

72. Kisser A, Zechmeister-Koss I. A systematic review of p16/Ki-67 immuno-testing for triage of low grade cervical cytology. BJOG. 2015;122:64-70.

73. Arbyn M, Kyrgiou M, Simoens C, et al. Perinatal mortality and other severe adverse pregnancy outcomes associated with treatment of cervical intraepithelial neoplasia: meta-analysis. BMJ. 2008;337:a1284.

74. Berenson $\mathrm{AB}$, Hirth JM, Chang $\mathrm{M}$. Change in human papillomavirus prevalence among US women aged 18-59 years, 2009-2014. Obstet Gynecol. 2017;130:693-701.

75. Wheeler CM, Skinner SR, Del Rosario-Raymundo MR, et al. Efficacy, safety, and immunogenicity of the human papillomavirus 16/18 AS04-adjuvanted vaccine in women older than 25 years: 7-year follow-up of the phase 3, double-blind, randomised controlled VIVIANE study. Lancet Infect Dis. 2016;16:1154-68.

76. Møller H, Gildea C, Meechan D, et al. Use of the English urgent referral pathway for suspected cancer and mortality in patients with cancer: cohort study. BMJ. 2015;351:h5102. 